\title{
乌s \\ Analysis of x-ray emission and electron dynamics in a capillary-guided laser wakefield accelerator
}

\author{
J. Ju, ${ }^{1}$ G. Genoud, ${ }^{2}$ H. E. Ferrari, ${ }^{3}$ O. Dadoun, ${ }^{4}$ B. Paradkar, ${ }^{1}$ K. Svensson, ${ }^{2}$ F. Wojda, ${ }^{2}$ M. Burza, ${ }^{2}$ \\ A. Persson, ${ }^{2}$ O. Lundh, ${ }^{2}$ N.E. Andreev, ${ }^{5}$ C.-G. Wahlström, ${ }^{2}$ and B. Cros ${ }^{1, *}$ \\ ${ }^{1}$ Laboratoire de Physique des Gaz et des Plasmas, CNRS-Université Paris-Sud, 91405, Orsay, France \\ ${ }^{2}$ Department of Physics, Lund University, P.O. Box 118, S-22100 Lund, Sweden \\ ${ }^{3}$ Consejo Nacional de Investigaciones Científicas y Técnicas (CONICET), 8400 Bariloche, Argentina \\ ${ }^{4}$ Laboratoire de l'Accélérateur Linéaire, CNRS-Université Paris-Sud, 91405, Orsay, France \\ ${ }^{5}$ Joint Institute for High Temperatures, Russian Academy of Sciences, Moscow 125412, Russia
}

(Received 30 January 2014; published 19 May 2014)

\begin{abstract}
The dynamics of electron acceleration driven by laser wakefield inside a $30.5 \mathrm{~mm}$ long dielectric capillary tube is analyzed using radiation emitted in the x-ray range. 3D particle-in-cell simulations, performed with parameters close to the experimental ones, show that in long plasmas, the accelerated electrons catch up and finally overrun the driving laser owing to a higher velocity of the electrons in the plasma. The electrons are then transversely scattered by the laser pulse, and penetrate the capillary wall where they generate bremsstrahlung radiation, modeled using GEANT4 simulations. The signature of bremsstrahlung radiation is detected using an x-ray camera, together with the betatron radiation emitted during electron acceleration in the plasma bubble. The reflection of betatron radiation from the inner capillary surface also accounts for a fraction of the observed signal on the x-ray camera. The simulation results are in agreement with the experimental ones and provide a detailed description of the electron and radiation properties, useful for the design of laser wakefield accelerators or radiation sources using long plasma media.
\end{abstract}

DOI: 10.1103/PhysRevSTAB.17.051302

PACS numbers: 52.38.Kd, 52.38.Ph, 52.65.Rr

\section{INTRODUCTION}

Charged particle accelerators play an important role in the development of modern science and technology, as the produced high energy particle beams are essential for several domains of fundamental and applied research. The dissemination of radio-frequency (rf) accelerators is mainly limited by their cost, induced by the large size of the facility required to produce $\mathrm{GeV}$ electron beams, as the magnitude of accelerating electric field inside a rf cavity is limited by material breakdown to be less than $\sim 100 \mathrm{MV} / \mathrm{m}$. Alternatively, in the mechanism of laser wakefield acceleration (LWFA) [1,2] electrons can be accelerated by the ultra-high electric fields, typically a few hundreds of $\mathrm{GV} / \mathrm{m}$, associated with a plasma wave. It has been demonstrated that LWFA is capable of producing high energy electrons of the order of hundred $\mathrm{MeV}$ over a few millimeters [3-5].

In LWFA, plasma waves are excited by the ponderomotive force of a short, intense laser pulse, which expels electrons out of high laser intensity regions. In the

\footnotetext{
"brigitte.cros@u-psud.fr

Published by the American Physical Society under the terms of the Creative Commons Attribution 3.0 License. Further distribution of this work must maintain attribution to the author $(s)$ and the published article's title, journal citation, and DOI.
}

nonlinear regime, plasma electrons can be completely expelled out of the intense laser volume and self-trapped in the accelerating potential of the plasma wave. Inside the bubblelike [6] accelerating cavity, electrons experience both longitudinal and transverse fields. Thus, these electrons can undergo strong transverse oscillations, known as betatron oscillations, giving rise to the emission of synchrotronlike radiation (called betatron radiation) [7-9], which has been observed and characterized in experiments [10-12].

Betatron radiation typically produces photons in the 1-10 keV range, and constitutes an attractive source for applications to time-resolved imaging due to its compactness and its intrinsic properties as it is brilliant, with duration as short as a few femtoseconds, and perfectly synchronized to the pump laser [11]. The x-ray properties depend on the accelerated electron energy, oscillation amplitude, betatron frequency, and through these quantities on the plasma density. Betatron radiation is thus an excellent diagnostic of the electron dynamics in LWFA. To increase the x-ray photon fluence and energy, electron energy and betatron amplitude can be maximized for a given plasma density. In order to achieve GeV-class electron beams [13] or betatron radiation with photon energy of $\sim 100 \mathrm{keV}$ or above [14], a few centimeter long plasma channels have been employed. The accelerated electron bunch has been observed to catch up with the laser 
tail in a millimeter-scale gas jet [15], or to propagate beyond the bubble center and interact with the laser pulse [14]. At high electron energy, when the laser frequency is a harmonic of the betatron frequency, a resonance occurs [16] and leads to a significant increase in the photon energy: for $700 \mathrm{MeV}$ electron maximum energy, photons energies in the range 1-7 MeV have been measured [14].

Using femtosecond laser pulses of 16 TW peak power, we have shown that the $\mathrm{x}$-ray peak brightness can be multiplied by a factor 30 when the laser beam is guided by a $10 \mathrm{~mm}$ long capillary tube instead of using a $2 \mathrm{~mm}$ long gas jet [17]. Electron bunches with tens of $\mathrm{pC}$ charge were measured to be accelerated to an energy up to $300 \mathrm{MeV}$, accompanied by x-ray emission with a peak brightness of the order of $10^{21} \mathrm{ph} / \mathrm{s} / \mathrm{mm}^{2} / \mathrm{mrad}^{2} / 0.1 \% \mathrm{BW}$. The $\mathrm{x}$-ray profile is geometrically related to the emission positions in the plasma by the rim of the capillary exit and provides a good probe of the acceleration process $[12,18]$. Using the emitted x-ray beam distribution to map the acceleration process, the number of betatron oscillations performed by accelerated electrons was inferred from the correlation between measured x-ray fluence and beam charge.

In this paper, we report on the analysis of the radiation emitted during the acceleration of an electron bunch by LWFA in a plasma long enough for the electron bunch to catch up with the laser pulse. The use of a capillary tube [19] to confine the gas and laser energy allows operation at arbitrary low plasma density and laser plasma interaction over long distances $[12,20]$. Electron acceleration in a $30.5 \mathrm{~mm}$ long capillary tube, where electrons are selftrapped and accelerated in the nonlinear bubble regime, and the associated radiation are analyzed. It is found that the trapped electrons are first accelerated and wiggled in the plasma bubble, producing betatron radiation, and furthermore the accelerated electrons catch up and overrun the driving laser pulse, a process during which electrons are scattered transversely by the laser ponderomotive force. Consequently, some of the accelerated electrons acquire a large transverse momentum and enter the downstream capillary wall, where they produce bremsstrahlung radiation. Numerical simulations of the electron acceleration process and radiation generation are performed to support the analysis of experimental observations.

\section{EXPERIMENT}

\section{A. Setup}

The experiment was conducted at the Lund Laser Centre (LLC) with the $10 \mathrm{~Hz}$ multi-terawatt Ti:Sapphire laser with a central wavelength of $\lambda_{0}=795 \mathrm{~nm}$, which delivered laser pulses of about $650 \mathrm{~mJ}$ on target within $40 \mathrm{fs}$ duration (FWHM). The experimental arrangement is schematically illustrated in Fig. 1. The laser was focused inside capillary tubes, $1 \mathrm{~mm}$ after the entrance, using an off-axis parabolic mirror with focal length $f=76 \mathrm{~cm}$. The laser focal

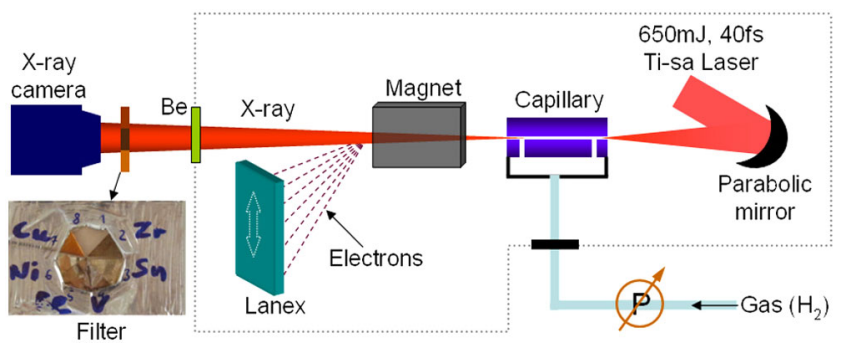

FIG. 1. Schematic illustration of the experimental setup. Elements within the dotted lines were in vacuum. The "pizza" filter consists of six different metals: $\mathrm{Zr}(3 \mu \mathrm{m}), \mathrm{Sn}(3 \mu \mathrm{m}), \mathrm{V}(3 \mu \mathrm{m})$, $\mathrm{Fe}(3 \mu \mathrm{m}), \mathrm{Ni}(5 \mu \mathrm{m}), \mathrm{Cu}(100 \mu \mathrm{m})$. All these metal pieces are attached onto a copper mesh together with a tin foil base.

spot was Airy-like with a radius at first minimum of $19.7 \pm 0.8 \mu \mathrm{m}$, and a peak intensity of $5.4 \times$ $10^{18} \mathrm{~W} / \mathrm{cm}^{2}$, corresponding to a normalized vector potential $a_{0} \simeq 1$.6. A five-degree-of-freedom mounted motorized housing was used to align capillary tubes. Hydrogen gas was filled into the capillary tubes through two approximately $270 \mu \mathrm{m}$ wide slits situated at $2.5 \mathrm{~mm}$ from the capillary ends [21]. To record electron spectra, a $10 \mathrm{~cm}$ long permanent magnet with central field of $0.7 \mathrm{~T}$ deflected the electrons onto a scintillating screen (Kodak Lanex Regular) imaged by a 12 bit charge coupled device (CCD) camera (not shown in Fig. 1). An aluminium plate was inserted in front of the Lanex screen to avoid its direct exposure to laser light. Electrons with energy less than $42 \mathrm{MeV}$ could not reach the Lanex screen, and therefore not be recorded. A 16 bit cooled CCD camera was placed in air about $110 \mathrm{~cm}$ away from the capillary end to record x-ray radiation exiting the vacuum chamber through two $150 \mu \mathrm{m}$ thick beryllium windows. The camera chip size was $13 \times 13 \mathrm{~mm}^{2}$, corresponding to a collecting solid angle of $12 \times 12 \mathrm{mrad}^{2}$. A so-called "pizza" filter, composed of six sector-shaped pieces of different metal foils, was placed in the beam to determine the photon energy.

\section{B. Results}

Using capillary tubes with a diameter of $178 \mu \mathrm{m}$, and length in the range from 10 to $30.5 \mathrm{~mm}$, electrons and $\mathrm{x}$-rays were observed to depend strongly on the plasma density, and the x-ray fluence was maximized when the plasma density was $n_{e}=(8.1 \pm 0.5) \times 10^{18} \mathrm{~cm}^{-3}$. For this value of density, electrons exhibit a Maxwellian-like spectral distribution with a cutoff energy as high as $200 \mathrm{MeV}$ and a beam charge of approximately $135 \mathrm{pC}$ [22]. Meanwhile, the accelerated electrons oscillate transversely at the betatron frequency $\omega_{\beta} \simeq \omega_{p} / \sqrt{2 \gamma}$ due to the focusing force inside the plasma bubble, where $\omega_{p}$ and $\gamma$ represent the plasma frequency and the relativistic factor, respectively. An electron with transverse oscillation amplitude $r_{0}$ generates electromagnetic radiation whose spectrum is determined by the wiggler strength parameter 


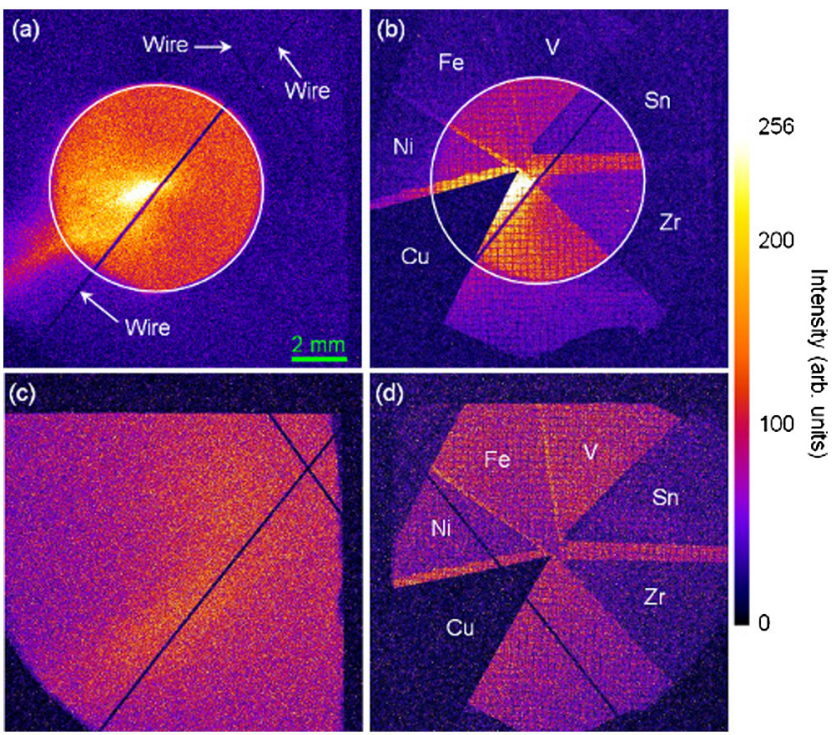

FIG. 2. Far-field X-ray images with or without the filter observed from capillary tubes with diameter of $178 \mu \mathrm{m}$ and different lengths: (a)-(b) $30.5 \mathrm{~mm}$, (c)-(d) $10 \mathrm{~mm}$ for a plasma density $(8.1 \pm 0.5) \times 10^{18} \mathrm{~cm}^{-3}$. There was a stainless steel wire $(\phi=50 \mu \mathrm{m})$ cross placed in the radiation beam path $53 \mathrm{~cm}$ away from the capillary exit, which is clearly resolved by the secondary emission in (a). The white circles in (a)-(b) highlight the shadows of the capillary exit rim. Each subfigure has a size of $13 \times 13 \mathrm{~mm}^{2}$, and corresponds to one laser shot.

$K_{\beta}=\gamma r_{0} \omega_{\beta} / c$, where $c$ denotes the speed of light in vacuum. In our experimental condition $K_{\beta} \gg 1$, the emission spectrum is synchrotron-like [7].

Figure 2 shows far-field $x$-ray images recorded by the x-ray camera for a plasma density of $(8.1 \pm 0.5) \times$ $10^{18} \mathrm{~cm}^{-3}$ using a $30.5 \mathrm{~mm}$ long capillary tube [(a) and (b)] and a $10 \mathrm{~mm}$ long tube in (c) and (d). Each image corresponds to one laser shot. In Figs. 2(b) and (d), the filter set was used in front of the camera and different transmission regions can be clearly observed; in Fig. 2(d), the circular structure near the edges of the image is due to the filter holder blocking the edges of the beam. Clear shadows of the capillary exit rim, highlighted by white circles in Figs. 2(a) and (b) are observed owing to cropping of the betatron emission by the capillary walls. By analyzing the $\mathrm{x}$-ray distribution at the edge of the beam displayed in Fig. 2, the process of betatron radiation and electron acceleration were determined [22] to start from $z \simeq$ $2 \mathrm{~mm}$ and extend to $z \simeq 7 \mathrm{~mm}, z=0$ corresponding to position of the capillary entrance on the longitudinal axis. In addition to betatron radiation inside the capillary rim, radiation can also be observed outside the white circle in Figs. 2(a) and (b). A cross made of stainless steel wires with a diameter of $50 \mu \mathrm{m}$ was placed in the optical path approximately $53 \mathrm{~cm}$ after the capillary tube and about $57 \mathrm{~cm}$ from the $\mathrm{x}$-ray camera. The shadows of the stainless steel wires, indicated by the arrows in Fig. 2(a), are clearly resolved even outside the rim of the capillary tube, providing evidence of another x-ray source illuminating the wires. Indeed, assuming x-ray sources located only in the range $z=2-7 \mathrm{~mm}$ inside the capillary tube, this region should be completely dark. This secondary emission can also be seen in the image with the filter [Fig. 2(b)], where there is still a detectable signal on the CCD camera behind the metal filters outside the shadow of the capillary exit rim. Without a secondary emission, the local signals through those metals would be as low as the noise level given by the copper part which blocks the secondary emission as well. This secondary emission was also detected with $20 \mathrm{~mm}$ long capillary tubes, but not as strong as with the $30.5 \mathrm{~mm}$ long capillary tube, and never with the $10 \mathrm{~mm}$ long tubes, which means the secondary emission depends on the capillary length. For the $10 \mathrm{~mm}$ long capillary tube shown in Figs. 2(c)-(d), roughly a quarter of the capillary shadow was recorded as the $\mathrm{x}$-ray divergence is larger because the $\mathrm{x}$-ray source is closer to the exit for these shorter capillary tubes. Before discussing in Sec. III the generation of secondary emission in long capillary tubes, we first examine the possibility for $\mathrm{x}$-rays generated by betatron oscillations to be reflected from the inner capillary wall.

\section{Discussion of x-ray reflection}

The roughness of the inner wall of the capillary used in this experiment is known to be smaller than $100 \mathrm{~nm}$ as the driving laser has been measured to be guided in matched capillary tubes according to theoretical predictions [19]. As the roughness of similar capillary tubes has been measured by Li et al. [23] to be of the order of $1 \mathrm{~nm}$, we evaluate in this section the fraction of the generated betatron radiation that would be reflected by a smooth capillary wall in our experimental conditions.

For clarity, the radiation detected inside the shadow of the capillary tube rim [i.e., inside the white circle in Figs. 2(a) and (b)] is called the main emission, as it exhibits a higher intensity. As radiation is detected for an angle larger than the cropping angle of the capillary exit, it could be envisaged that part of the radiation is reflected by the capillary inner surface and goes out of the capillary tube as a secondary emission, as illustrated in Fig. 3.

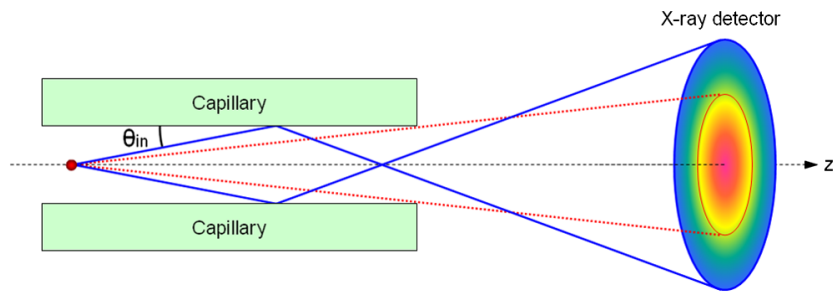

FIG. 3. Schematic illustration of x-rays cropped or reflected by the capillary tube. The cropped part confined within the two dotted lines is the main emission, and the solid lines indicate $\mathrm{x}$-rays that would be reflected by the capillary inner surface to produce a secondary emission on the detector. 
The x-ray intensity distribution in the detector plane, taking into account $\mathrm{x}$-ray reflection, can be estimated as follows. The intensity of betatron radiation produced by a wiggling relativistic electron is given by [7]

$$
\begin{aligned}
\frac{d^{2} I}{d E d \Omega} \simeq & \frac{3 e^{2}}{2 \pi^{3} \varepsilon_{0} c} \gamma^{2} \xi^{2}\left(1+\gamma^{2} \theta^{2}\right) \\
& \times\left[\frac{\gamma^{2} \theta^{2}}{1+\gamma^{2} \theta^{2}} \mathcal{K}_{1 / 3}^{2}(\xi)+\mathcal{K}_{2 / 3}^{2}(\xi)\right],
\end{aligned}
$$

where $E$ denotes the photon energy, $\varepsilon_{0}$ is the permittivity of free space, and $\xi=\left(1+\gamma^{2} \theta^{2}\right)^{3 / 2} E / E_{c}$ with $E_{c}[\mathrm{keV}]=$ $1.1 \times 10^{-23} \gamma^{2} n_{e} r_{0}$ representing the critical energy. $\theta$ is the emission angle with respect to the longitudinal axis, equal to the incident angle $\theta_{\text {in }}$ defined in Fig. 3. $\mathcal{K}_{1 / 3}$ and $\mathcal{K}_{2 / 3}$ are modified Bessel functions of order $1 / 3$ and $2 / 3$, respectively. At a given longitudinal position $z$ inside the capillary tube, one can geometrically calculate the radial location on the detector, $r\left(z, \theta_{\text {in }}\right)$, where the radiation will be received, and evaluate the corresponding CCD counts at $r\left(z, \theta_{\text {in }}\right)$ by

$$
C(r)=\int_{z_{1}}^{z_{2}} \int_{E_{1}}^{E_{2}} \frac{d^{2} I}{d E d \Omega} R_{X} T_{\mathrm{air}} T_{\mathrm{Be}} Q_{\mathrm{ccd}} \frac{E}{G_{\mathrm{ccd}}} d E d z
$$

where $z_{1}=2 \mathrm{~mm}, z_{2}=7 \mathrm{~mm}$ represent the longitudinal positions limiting the source of betatron radiation inside the capillary tube [22], $Q_{\text {ccd }}, G_{\text {ccd }}$ denote quantum efficiency and gain of the x-ray CCD camera, $T_{\text {air }}$ and $T_{\mathrm{Be}}$ are x-ray transmissions through the $5 \mathrm{~mm}$ air gap and $300 \mu \mathrm{m}$ thick beryllium windows in the optical path, respectively. $R_{X}$ represents the X-ray reflectivity on the inner capillary wall, which depends on photon energy, incident angle, and roughness of inner capillary wall.

Considering the x-ray CCD quantum efficiency, $E_{1}, E_{2}$ were set to be 0.1 and $20 \mathrm{keV}$, respectively. The evolution of the gamma factor $\gamma(z)$ obtained from 3D PIC simulation [22] and the betatron oscillation amplitude $r_{0}=2 \mu \mathrm{m}$ found in experiment [17] were used to calculate Eq. (2). Figure 4(a) gives the $\mathrm{x}$-ray reflectivity [24] for different surface roughness when the incident angle $\theta_{\text {in }}=4.5 \mathrm{mrad}$,
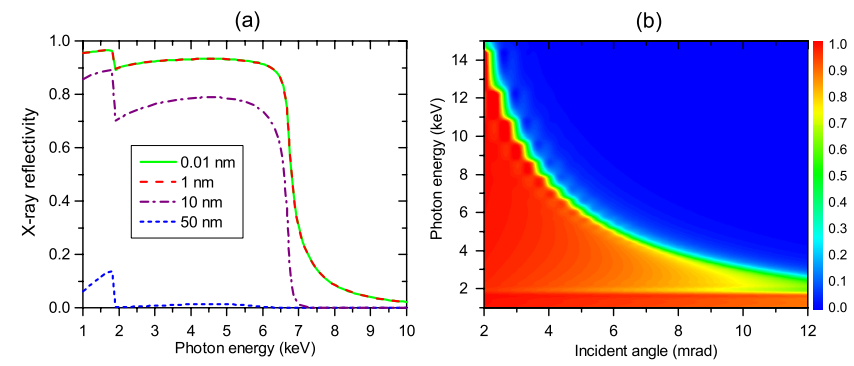

FIG. 4. (a) x-ray reflectivity as a function of photon energy for different surface roughness when $\theta_{\text {in }}=4.5 \mathrm{mrad}$, and (b) x-ray reflectivity as a function of incident angle and photon energy for a surface roughness of $1 \mathrm{~nm}$. assuming the capillary tube is made of pure $\mathrm{SiO}_{2}$. It shows that x-ray reflectivity drops for increasing surface roughness, and does not appreciably change for wall roughness smaller then $1 \mathrm{~nm}$, whereas it becomes negligible for roughness larger than $50 \mathrm{~nm}$. Figure 4(b) shows the x-ray reflectivity for a surface roughness of $1 \mathrm{~nm}$ as a function of incident angle $\theta_{\text {in }}$ and photon energy $E$, which was used to calculate $C(r)$ in Eq. (2). If the incident angle $\theta_{\text {in }}$ is so small that betatron radiation leaves the capillary tube without reflection, $R_{X}=1$.

Figure 5(a) illustrates the X-ray pattern in the detector plane calculated numerically when the $\mathrm{x}$-ray reflection at the inner wall of the capillary with roughness of $1 \mathrm{~nm}$ is taken into account as explained above. For comparison, Fig. 5(b) displays the numerical result when there is no $\mathrm{x}$-ray reflection, namely photons hitting the capillary wall are lost; Figs. 5(c) and (d) are the lineout intensity profiles corresponding to $y=0$ in Figs. 5(a) and (b), respectively. A sharp shadow of the capillary exit rim is produced and the $\mathrm{x}$-ray beam is cropped in the absence of x-ray reflection [Figs. 5(b), (d)], whereas the rim shadow becomes blurred when $\mathrm{x}$-ray reflection is taken into account. It suggests that $\mathrm{x}$-ray reflection could contribute to the secondary emission shown in Figs. 2(a)-(b). However, the intensity patterns in Fig. 5 and in Fig. 2(a) are significantly different, as the results shown in Fig. 5 are obtained from a single-electron radiation model, whereas the experimental patterns are generated by a distribution of electrons with significant energy spread and spatial extension. In order to perform a quantitative comparison, we select a ring with radius $r=5 \mathrm{~mm}$, located outside the rim shadow in Fig. 2(a), and where the x-ray reflection mainly modifies the profile in Fig. 5. According to Fig. 5(a), the normalized CCD count at $r=5 \mathrm{~mm}$ is about 0.07 . The peak CCD count observed experimentally in Fig. 2(a) is around 3000, so the corresponding CCD count at $r=5 \mathrm{~mm}$ can be estimated to be
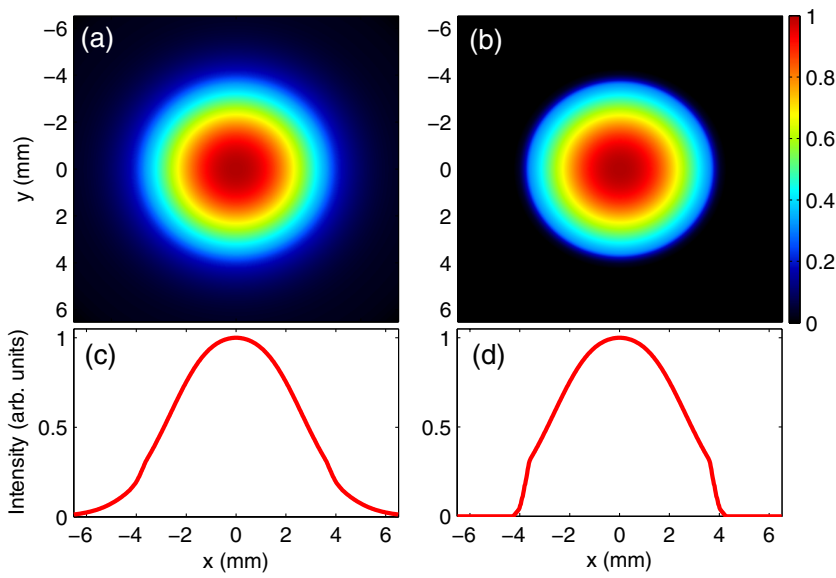

FIG. 5. Numerically calculated far-field x-ray patterns in the detector plane when (a) x-ray reflection was taken into account, (b) no x-ray reflection was considered. (c)-(d) are the corresponding intensity profiles through $y=0$. 
$3000 \times 0.07=210$. However for the case of Fig. 2(a), $410 \pm 60 \mathrm{CCD}$ counts were typically measured at $r=5 \mathrm{~mm}$, which is about twice higher than this numerical prediction. It implies that besides $\mathrm{x}$-ray reflection some other mechanism contributes to the formation of the secondary emission.

\section{SIMULATION OF ELECTRON DYNAMICS}

As $30.5 \mathrm{~mm}$ long plasmas are longer than the typical acceleration distance and laser depletion length for the parameters of our experiment, the electron dynamics during the acceleration process has to be investigated in detail. To do so, 3D particle-in-cell (PIC) simulations were performed with the code CALDER-CIRC [25] including the boundary condition of capillary tube described in Ref. [26]. The simulation parameters are set similar to the experimental ones. To shorten the time for parameter scan in 3D PIC simulations, a smaller capillary tube diameter of $152 \mu \mathrm{m}$ was used. It was verified in simulation that the laser-plasma interaction is nearly the same for capillary tubes with 152 and $178 \mu \mathrm{m}$ diameters owing to a relatively small laser focal spot. The laser pulse with $a_{0}=1.6$ and a FWHM pulse duration of $40 \mathrm{fs}$ was focused $1 \mathrm{~mm}$ inside the capillary tube. A density plateau $n_{e}=8 \times 10^{18} \mathrm{~cm}^{-3}$ is set between the two capillary slits; outside the plateau, the density fall is modeled as a $3 \mathrm{~mm}$ long linear density ramp decreasing from $n_{e}$ to 0 [22]. Ten particles per cell, spatial mesh sizes $d z=\lambda_{0} /(8 \pi), d r=\lambda_{0} / \pi$ were used in the simulations.

Figure 6 shows one simulation case for a plasma density $n_{e}=8 \times 10^{18} \mathrm{~cm}^{-3}$. In Fig. 6(a), a plasma bubble is produced behind the laser and starts trapping electrons at $z \simeq 3.195 \mathrm{~mm}$. Afterwards, the accelerated electrons catch up with the laser tail and get scattered by the laser ponderomotive force. The increase in transverse size of the electron bunch can be seen in Fig. 6(c). The distance that it takes for a trapped electron (moving at nearly the speed of light $c$ ) to catch up with the driving laser can be approximated by $L=R_{b} c /\left(c-v_{g}\right)$. The bubble radius in
Fig. 6(b) is $R_{b} \simeq 7 \mu \mathrm{m}$, and the laser group velocity is given by $v_{g}=c \omega_{0}\left(\omega_{0}^{2}+\omega_{p}^{2}\right)^{-1 / 2} \simeq 0.997 c$, which yields $L \simeq 2.3 \mathrm{~mm}$. The laser pump depletion length can be evaluated [27] through $L_{p d}=c \tau_{0} \omega_{0}^{2} / \omega_{p}^{2}$ to be $L_{p d} \simeq$ $3 \mathrm{~mm}$, where $\tau_{0}$ denotes the laser pulse duration. Since $L$ is comparable with $L_{p d}$, some electrons can catch up with the laser before the laser is depleted. During laser depletion, a fraction of the pulse propagates backward and interacts with the trailing electron bunch as can be seen in Fig. 6(c).

Figure 7 shows the distribution of the electron bunch in the transverse plane at different longitudinal positions, namely $z=4.11,5.87$, and $7.63 \mathrm{~mm}$. The simulation parameters are the same as for Fig. 6. A tiny bright spot can be seen in Fig. 7(a), corresponding to the typical electron bunch distribution when it catches up with the driving laser. The bunch size is dramatically enlarged after interacting with the laser pulse [Fig. 7(b)], and afterwards the outmost electrons start reaching and entering into the capillary wall. Owing to a larger emittance of the electron bunch along the laser polarization direction [15], the bunch explosion is more pronounced in that direction as shown in Figs. 7(b)-(c). Figures 7(d)-(e) display typical trajectories of some electrons which are strongly scattered. Since the ponderomotive force does not depend on laser polarization, the electrons are expelled both along and perpendicular to the laser polarization direction.

In summary, PIC simulations show that in our experimental conditions, the accelerated electrons catch up with the laser pulse and are scattered when they overrun it. As a result, the electrons reach the capillary tube wall, where they produce bremsstrahlung radiation. It should be mentioned that the capillary radius used in the experiments $(89 \mu \mathrm{m})$ is larger than that of the simulation case $(76 \mu \mathrm{m})$, so the outmost scattered electrons should reach the real capillary wall at a longitudinal position downstream the case of Fig. 7(c) $z=7.63 \mathrm{~mm}$. Therefore only a few electrons are likely to reach the capillary wall before $z=10 \mathrm{~mm}$, which can explain why no detectable secondary emission was found in experiment with $10 \mathrm{~mm}$ long
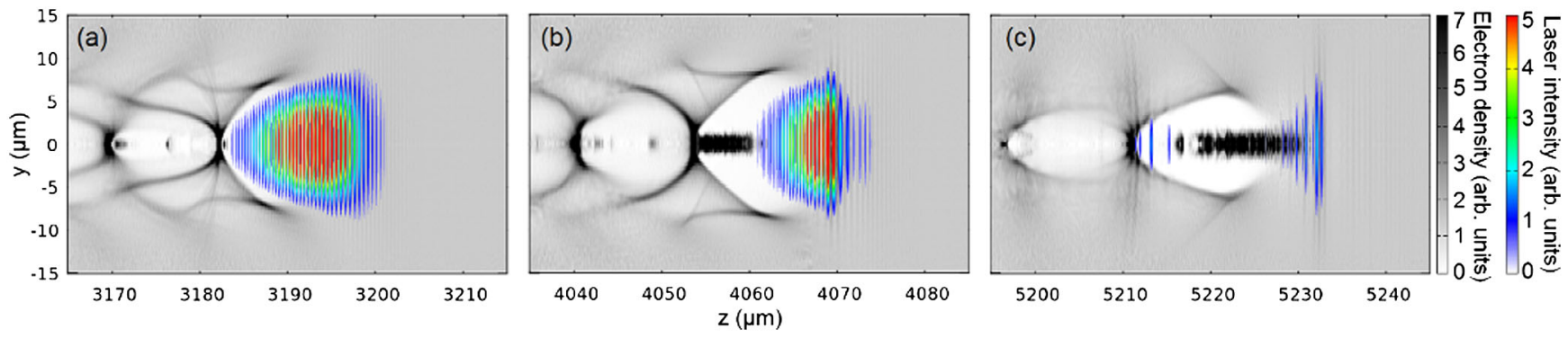

FIG. 6. Snapshots of plasma density (gray scale) and instantaneous laser intensity (color scale), in which the laser propagates from left to right at three different positions along the axis of capillary tube. Electron trapping starts at $z \simeq 3.195 \mathrm{~mm}$ in (a), and at $z \simeq 4.065 \mathrm{~mm}$ (b) a large amount of electrons are trapped and accelerated in the first plasma bubble, where the head of the electron bunch catches up with the laser. The electrons interact with the laser and get modulated at $z \simeq 5.225 \mathrm{~mm}(\mathrm{c})$, where the transverse size of the electron bunch is appreciably increased. 

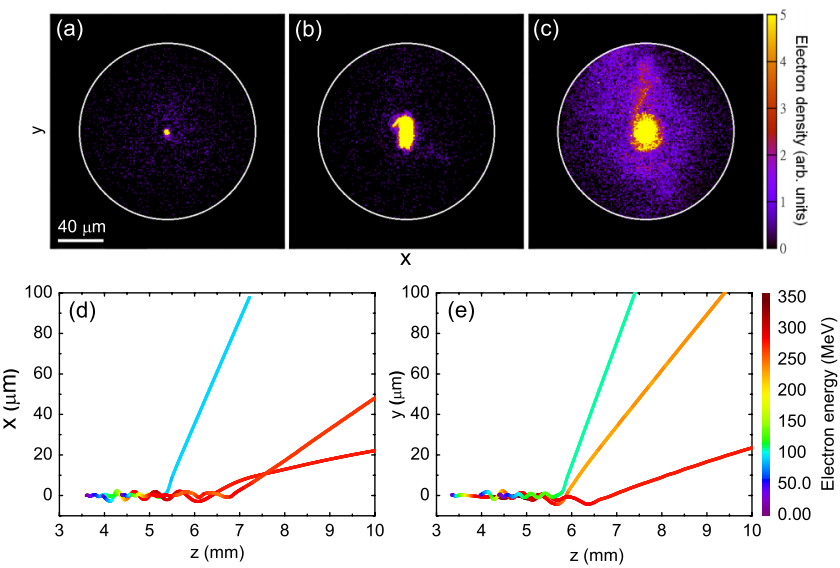

FIG. 7. Electron distribution in the transverse plane at different longitudinal positions (a) $4.11 \mathrm{~mm}$, (b) $5.87 \mathrm{~mm}$, and (c) $7.63 \mathrm{~mm}$, where the circle indicates the capillary border of $152 \mu \mathrm{m}$ diameter. The increase in electron beam size is clearly visible. The laser is polarized along the vertical ( $y$ ) axis. Typical electron trajectories in the planes (d) perpendicular and (e) parallel with the laser polarization. The simulation parameters are the same as for Fig. 6.

capillary tubes. For longer capillary tubes, more electrons interact with the capillary wall, which explains why secondary emission was detected for $20 \mathrm{~mm}$ long capillary tubes, and became much stronger for the $30.5 \mathrm{~mm}$ long tube in our experiments. In order to evaluate the amplitude of this secondary emission, we have modeled the generation of bremsstrahlung radiation by the electron distribution obtained from PIC simulations.

\section{MODELING OF BREMSSTRAHLUNG RADIATION}

In order to determine the amount of secondary emission shown in Figs. 2(a)-(b) that could be produced by bremsstrahlung radiation, we have calculated the spectrum and angular distribution of bremsstrahlung radiation, and evaluated the signal generated on the $\mathrm{x}$-ray camera, sensitive to 1-20 keV photons and with a collecting solid angle of $12 \times 12 \mathrm{mrad}^{2}$. The bremsstrahlung radiation produced by the electron bunch was calculated using the code GEANT4 [28], which simulates the passage of particles through matter using the Monte-Carlo method. Particle transport is described taking into account the processes of bremsstrahlung, multiple scattering, and ionization, while the produced electromagnetic radiation experiences the processes of photoelectric effect, Compton scattering, and conversion into electron-positron pair.

The bremsstrahlung modeling is initialized with the electron beam obtained from PIC simulation at $z=7.63 \mathrm{~mm}$, where the electron bunch has already been modulated after overrunning the laser pulse, but not yet reached the capillary wall. After $z=7.63 \mathrm{~mm}$, both experimental and PIC simulation results [22] show that

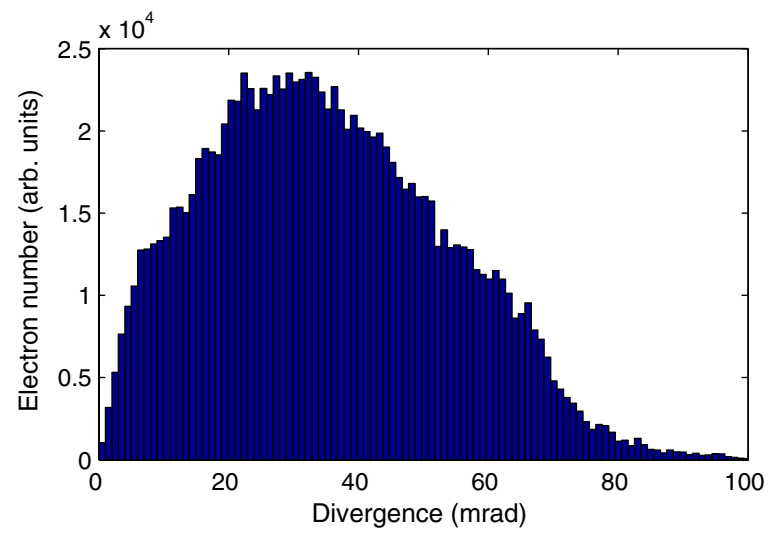

FIG. 8. Angular distribution of the electron bunch obtained from PIC simulation at $z=7.63 \mathrm{~mm}$.

the laser becomes depleted so that it cannot considerably modulate the electron bunch. The electron beam transverse distribution used in the GEANT4 simulation is shown in Fig. 7(c), and the corresponding beam divergence is shown in Fig. 8, where the divergence angle is defined as $\theta_{d}=$ $\arctan \left[\left(p_{x}^{2}+p_{y}^{2}\right)^{1 / 2} / p_{z}\right]$ with $p_{j}$ representing electron momentum along the $j$ axis. In the GEANT 4 calculation, $4 \times 10^{4}$ macroelectrons were used. The capillary tube, taken as pure $\mathrm{SiO}_{2}$, is $30.5 \mathrm{~mm}$ long with inner and outer radii of $89 \mu \mathrm{m}$ and $2.75 \mathrm{~mm}$, respectively. The two Be windows with total thickness of $300 \mu \mathrm{m}$ and the $5 \mathrm{~mm}$ air gap, as illustrated in Fig. 1, were included in the modeling as well.

Figure 9 shows the particle trajectories in the capillary wall, where the red, blue, and green lines represent the trajectories of electrons, positrons, and photons, respectively. It shows that numerous photons are produced by bremsstrahlung radiation when electrons pass through the capillary wall, and most of the photons move forward very directionally. The spectrum of the emitted photons and their angular distribution are given in Fig. 10. The overall spectrum [Fig. 10(a)] exponentially drops with only a few photons remaining beyond $200 \mathrm{MeV}$. Figure 10(c) shows the spectrum for photon energy below $500 \mathrm{keV}$, indicating the spectral intensity peaks around $20 \mathrm{keV}$, and diminishes when photon energy decreases toward zero.

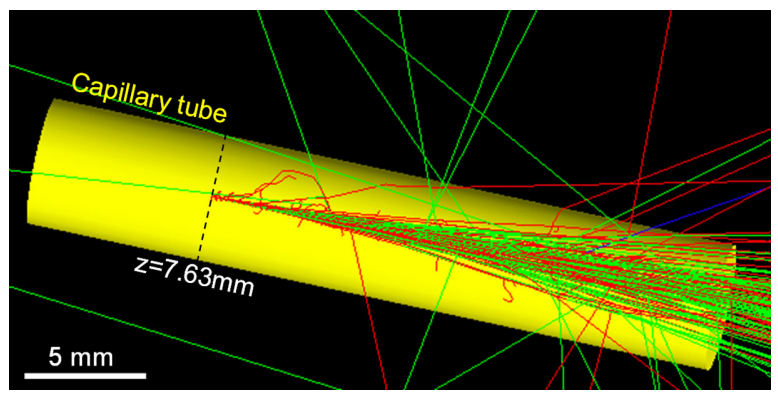

FIG. 9. Trajectories of 50 simulated particles in the capillary part (yellow) from the GEANT4 modeling: photons (green), electrons (red), and positrons (blue). 

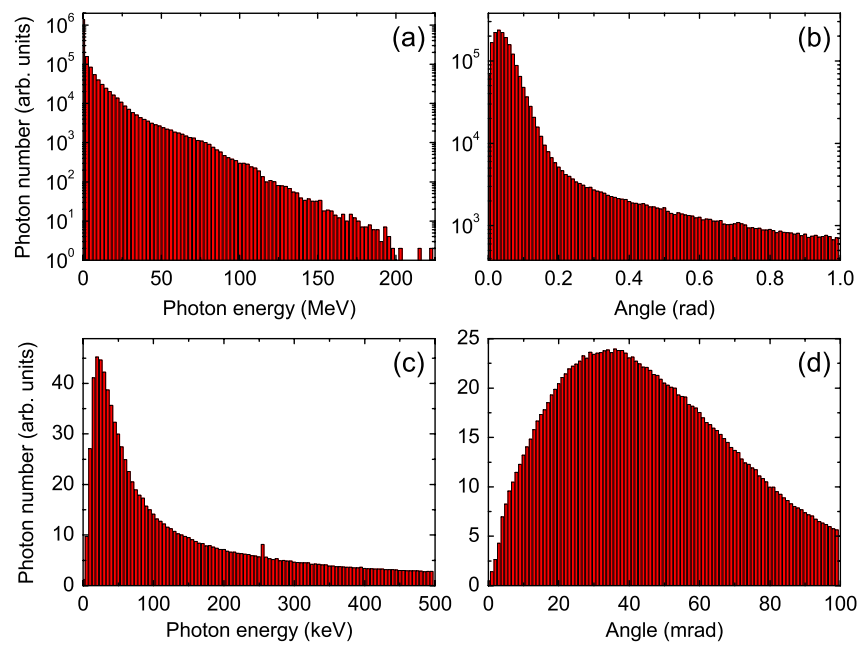

FIG. 10. (a) Spectrum and (b) angular distribution of the total bremsstrahlung radiation in logarithmic scale. (c) Spectrum for 0-500 keV photons and (d) angular distribution for 0-100 mrad.

Furthermore, most of the photons are collimated within a divergence cone of $100 \mathrm{mrad}$ as shown in Fig. 10(b). Particularly, a peak at $\theta \simeq 35 \mathrm{mrad}$ is observed in Fig. 10(d). This peak perfectly coincides with the divergence peak of the electron beam given in Fig. 8, implying the photons are indeed generated by the scattered electrons.

It is important to check whether this bremsstrahlung radiation could be detected by the x-ray camera and furthermore induce the x-ray halos observed in Fig. 2. First, the photon number generated by bremsstrahlung radiation needs to be estimated. Here we particularly consider the photons with a divergence of $4<\theta<5 \mathrm{mrad}$, corresponding to a ring of $4.5 \times 10^{4}$ pixels outside the capillary shadow (the white circle) in Fig. 2(a), where the signal could be induced by the bremsstrahlung photons. The charge of the electron bunch is $\sim 80 \mathrm{pC}$ in experiments [22], and the electron-to-photon $(4<\theta<6$ mrad) conversion efficiency is found to be $0.7 \%$ in the GEANT4 simulation, which yields $3.7 \times 10^{6}$ photons produced in the angle range of 4-5 mrad. The average fluence is therefore calculated to be approximately 80 photons per pixel. We shall furthermore estimate the resulting CCD counts. To do so, only the photons with energy less than $50 \mathrm{keV}$ were taken into account, because the detector responsivity for higher energy photons is negligible. Using the radiation spectrum obtained in the simulation and the quantum efficiency of the $\mathrm{x}$-ray camera, we estimate that this radiation could produce an average CCD count of around 270 for $4<\theta<5 \mathrm{mrad}$. It should be noted that the relatively low CCD count is due to the poor detector responsivity for high energy photons, and not to a low fluence of bremsstrahlung photons.

In summary, the above numerical calculations show that $\mathrm{x}$-ray reflection and bremsstrahlung radiation can together yield $480(210+270)$ CCD counts outside the shadow of the capillary border, which is consistent with the experimental observation of about $410 \pm 60 \mathrm{CCD}$ counts. We therefore conclude that $\mathrm{x}$-ray reflection and bremsstrahlung radiation both contribute to the secondary emission observed in experiments.

\section{CONCLUSION}

In conclusion, we analyzed the dynamics of electrons acceleration by laser wakefield inside a long plasma medium when the laser is guided by a dielectric capillary tube. It was found that the accelerated electrons are scattered when they catch up and overrun the driving laser pulse. This phenomenon is undesired for the generation of collimated beams of high energy electrons, and can be avoided by making the laser pump depletion length shorter than the dephasing length, so the laser becomes greatly damped before the electrons catch up with it. Betatron radiation is emitted over a distance of about $5 \mathrm{~mm}$ and generates a bright beam, with a peak brightness of the order of $10^{21} \mathrm{ph} / \mathrm{s} / \mathrm{mm}^{2} / \mathrm{mrad}^{2} / 0.1 \% \mathrm{BW}$, detected on the $\mathrm{x}$-ray CCD. In addition to betatron radiation detected inside the shadow of the capillary tube, the x-ray images exhibit the signature of secondary emission in the $\mathrm{x}$-ray range.

The detailed analysis of this secondary emission shows that it may be produced by two mechanisms: x-ray reflection from the inner capillary surface and bremsstrahlung radiation produced when scattered electrons propagate inside the wall of the capillary tube. It was not possible to discriminate the contributions of the two mechanisms in the present experimental configuration; the selective detection of photons with energy larger than $20 \mathrm{keV}$ should provide a clear signature of bremsstrahlung radiation in future work. According to simulations, $10^{8}-10^{9}$ photons per shot with photon energy extending up to $\sim 200 \mathrm{MeV}$ can be generated by bremsstrahlung radiation for an electron beam obtained in our experiments with a charge of the order of $100 \mathrm{pC}$. Such gamma-ray beams are very directional and as intense as those generated by schemes like Compton scattering [29], which can be applied to imaging the interior structure of dense objects [30].

\section{ACKNOWLEDGMENTS}

This work was funded by LASERLAB-EUROPE (Grant Agreement No. 228334), the Triangle de la Physique Contract No. 2011-086TMULTIPLACCELE, the Lund University x-ray Center (LUXC), the Swedish Research Council (including the Linné grant to LLC), the Swedish Foundation for Strategic Research (SSF), and the Knut and Alice Wallenberg Foundation. J. Ju acknowledges financial support from Chinese Scholarship Council (CSC).

[1] T. Tajima, and J. Dawson, Phys. Rev. Lett. 43, 267 (1979).

[2] E. Esarey, C. B. Schroeder, and W. P. Leemans, Rev. Mod. Phys. 81, 1229 (2009). 
[3] S. P. D. Mangles, C. D. Murphy, Z. Najmudin, A. G. R. Thomas, J. L. Collier, A. E. Dangor, E. J. Divall, P. S. Foster, J. G. Gallacher, C. J. Hooker, D. A. Jaroszynski, A. J. Langley, W. B. Mori, P. A. Norreys, F. S. Tsung, R. Viskup, B. R. Walton, and K. Krushelnick, Nature (London) 431, 535 (2004).

[4] C. G. R. Geddes, Cs. Toth, J. van Tilborg, E. Esarey, C. B. Schroeder, D. Bruhwiler, C. Nieter, J. Cary, and W. P. Leemans, Nature (London) 431, 538 (2004).

[5] J. Faure, Y. Glinec, A. Pukhov, S. Kiselev, S. Gordienko, E. Lefebvre, J. P. Rousseau, F. Burgy, and V. Malka, Nature (London) 431, 541 (2004).

[6] A. Pukhov, and J. Meyer-ter-Vehn, Appl. Phys. B 74, 355 (2002).

[7] E. Esarey, B. A. Shadwick, P. Catravas, and W. P. Leemans, Phys. Rev. E 65, 056505 (2002).

[8] A. G. R. Thomas, Phys. Plasmas 17, 056708 (2010).

[9] S. Corde, K. Ta Phuoc, G. Lambert, R. Fitour, V. Malka, A. Rousse, A. Beck, and E. Lefebvre, Rev. Mod. Phys. 85, 1 (2013).

[10] A. Rousse, K. Ta Phuoc, R. Shah, A. Pukhov, E. Lefebvre, V. Malka, S. Kiselev, F. Burgy, J. P. Rousseau, D. Umstadter, and D. Hulin, Phys. Rev. Lett. 93, 066403 (2004).

[11] S. Kneip, C. McGuffey, J. L. Martins, S. F. Martins, C. Bellei, V. Chvykov, F. Dollar, R. Fonseca, C. Huntington, G. Kalintchenko, A. Maksimchuk, S. P. D. Mangles, T. Matsuoka, S. R. Nagel, C. A. J. Palmer, J. Schreiber, K. Ta Phuoc, A. G. R. Thomas, V. Yanovsky, L. O. Silva, K. Krushelnick, and Z. Najmudin, Nat. Phys. 6, 980 (2010).

[12] G. Genoud, K. Cassou, F. Wojda, H. E. Ferrari, C. Kamperidis, M. Burza, A. Persson, J. Uhlig, S. Kneip, S. P. D. Mangles, A. Lifschitz, B. Cros, and C.-G. Wahlstrom, Appl. Phys. B 105, 309 (2011).

[13] W. P. Leemans, B. Nagler, A. J. Gonsalves, Cs. Tóth, K. Nakamura, C. G. R. Geddes, E. Esarey, C. B. Schroeder, and S. M. Hooker, Nat. Phys. 2, 696 (2006).

[14] S. Cipiccia, M. R. Islam, B. Ersfeld, R. P. Shanks, E. Brunetti, G. Vieux, X. Yang, R. C. Issac, S. M. Wiggins, G. H. Welsh, M.-P. Anania, D. Maneuski, R. Montgomery, G. Smith, M. Hoek, D. J. Hamilton, N. R. C. Lemos, D. Symes, P. P. Rajeev, V. O. Shea, J. M. Dias, and D. A. Jaroszynski, Nat. Phys. 7, 867 (2011).
[15] S. P. D. Mangles, A. G. R. Thomas, M. C. Kaluza, O. Lundh, F. Lindau, A. Persson, F. S. Tsung, Z. Najmudin, W. B. Mori, C.-G. Wahlstrom, and K. Krushelnick, Phys. Rev. Lett. 96, 215001 (2006).

[16] I. Nam, M. S. Hur, H. S. Uhm, N. A. M. Hafz, and H. Suk, Phys. Plasmas 18, 043107 (2011).

[17] J. Ju, K. Svensson, A. Dopp, H. E. Ferrari, K. Cassou, O. Neveu, G. Genoud, F. Wojda, M. Burza, A. Persson, O. Lundh, C.-G. Wahlstrom, and B. Cros, Appl. Phys. Lett. 100, 191106 (2012).

[18] S. Corde, C. Thaury, K. Ta Phuoc, A. Lifschitz, G. Lambert, J. Faure, O. Lundh, E. Benveniste, A. BenIsmail, L. Arantchuk, A. Marciniak, A. Stordeur, P. Brijesh, A. Rousse, A. Specka, and V. Malka, Phys. Rev. Lett. 107, 215004 (2011).

[19] B. Cros, C. Courtois, G. Matthieussent, A. Di Bernardo, D. Batani, N. Andreev, and S. Kuznetsov, Phys. Rev. E 65, 026405 (2002).

[20] H. E. Ferrari, A. F. Lifschitz, G. Maynard, and B. Cros, Phys. Plasmas 18, 083108 (2011).

[21] J. Ju, and B. Cros, J. Appl. Phys. 112, 113102 (2012).

[22] J. Ju, K. Svensson, H. Ferrari, A. Dopp, G. Genoud, F. Wojda, M. Burza, A. Persson, O. Lundh, C.-G. Wahlstrom, and B. Cros, Phys. Plasmas 20, 083106 (2013).

[23] Y.-D. Li, X.-Y. Lin, Z.-Y. Tan, T.-X. Sun, and Z.-G. Liu, Chin. Phys. B 20, 040702 (2011).

[24] The center for X-Ray optics, http://www.cxro.lbl.gov/.

[25] A. F. Lifschitz, X. Davoine, E. Lefebvre, J. Faure, C. Rechatin, and V. Malka, J. Comput. Phys. 228, 1803 (2009).

[26] H. E. Ferrari, A. Lifschitz, and B. Cros, Plasma Phys. Controlled Fusion 53, 014005 (2011).

[27] W. Lu, M. Tzoufras, C. Joshi, F. S. Tsung, W. B. Mori, J. Viera, R. A. Fonseca, and L. O. Silva, Phys. Rev. ST Accel. Beams 10, 061301 (2007).

[28] S. Agostinelli et al., Nucl. Instrum. Methods Phys. Res., Sect. A 506, 250 (2003).

[29] S. Chen, N. D. Powers, I. Ghebregziabher, C. M. Maharjan, C. Liu, G. Golovin, S. Banerjee, J. Zhang, N. Cunningham, A. Moorti, S. Clarke, S. Pozzi, and D. P. Umstadter, Phys. Rev. Lett. 110, 155003 (2013).

[30] A. Ben-Ismail, O. Lundh, C. Rechatin, J. K. Lim, J. Faure, S. Corde, and V. Malka, Appl. Phys. Lett. 98, 264101 (2011). 